\title{
Iron dependent erythropoiesis in women with excessive menstrual blood losses and women with normal menses
}

Mariasanta Napolitano, $\mathrm{MD}^{1}$; Alberto Dolce, $\mathrm{PhD}^{2}$; Giuseppe Celenza, $\mathrm{PhD}^{3}$; Elvira Grandone, $\mathrm{MD}^{4}$; Maria Grazia Perilli, $\mathrm{PhD}^{3}$; Sergio Siragusa, $\mathrm{MD}^{1}$; Gaspare Carta, $\mathrm{MD}^{5}$; Assunta Orecchioni, MD; ${ }^{6}$ and Guglielmo Mariani, $\mathrm{MD}^{7}$

1. Hematology Unit, Thrombosis and Hemostasis Reference Regional Center, University of Palermo, Palermo-Italy

2. Istituto Superiore Italiano di Statistica (ISTAT), Palermo, Italy

3. Biochemical Sciences Department, University of L'Aquila, L'Aquila-Italy

4. Thrombosis and Hemostatis Center, IRCCS Casa "Sollievo della Sofferenza", S. Giovanni Rotondo, Italy

5. Gynecology and Obstetric Unit, University of L'Aquila, L'Aquila-Italy

6. Clinical Pathology Unit, Central Laboratory of Thrombosis and Hemostasis, "S. Salvatore" Regional Hospital, L'Aquila-Italy

7. University of Ferrara, Ferrara-Italy

Corresponding author:

Mariasanta Napolitano, MD

Hematology Unit, Thrombosis and Hemostasis Reference Regional Center Policlinico "P. Giaccone"

Via del Vespro, 1-90100- Palermo- Italy

Tel: $+390916554405-4403$

Fax: +390916554002

E-mail: mariasanta.napolitano@unipa.it

Table of content: Normal hematopoiesis

Text word count: 2837 (References, Table anf Figure Legends excluded) Keywords: Iron losses, Iron deficiency, Iron deficient anemia, Menstrual blood losses 


\begin{abstract}
In women of fertile age, iron loss consequent to excessive menstrual discharge is by far the most frequent cause of iron-deficient anemia. However, the relationship between menstrual discharge and iron loss is poorly understood. In this prospective study, total menstrual and iron losses were assayed in a large cohort of non-anemic women and women with excessive menstrual blood losses (menorrhagia) in order to provide data useful for intervention. One hundred and five Caucasian women aged 20-45 years were recruited. Blood cell count and serum ferritin (SF) levels were determined in each case before menses. Menstrual Fluid Losses (MFL) were determined using a standardized pads' weight method. Hematin concentration was assayed by a variant of the Alkaline Hematin Method from which iron concentration was calculated. Mean SF levels were 36.2 (range 8.6-100) $\mathrm{ng} / \mathrm{ml}$ in healthy women and 6.4 (range 5-14) $\mathrm{ng} / \mathrm{ml}$ in patients with menorrhagia. Median values of iron lost/cycle were $0.87 \mathrm{mg}$ in healthy women and $5.2 \mathrm{mg}$ in patients with menorrhagia (ranges: 0.102-2.569 and 1.634-8.665 $\mathrm{mg}$, respectively, $\mathrm{p}<0.001$ ). In women with menorrhagia, iron lost/cycle strongly correlated $(0.789, \mathrm{p}<0.001)$ with MFL. In conclusion, healthy women with normal menses lose, on average, $1 \mathrm{mg}$ iron/cycle. Average iron losses in patients with menorrhagia are, at least in our cohort, on average, 5-6 times higher than normal. Most women with menorrhagia had totally depleted iron stores. Indirect, quantitative evaluation of iron lost with menses may be useful to assess the risk of developing iron-deficient anemia in individual patients.
\end{abstract}




\section{Introduction}

Iron is a vital element of metabolism, with the most obvious and known role played in erythropoiesis, and important functions in other intracellular processes in all tissues of the body. The uptake of iron is finely regulated (1). For physiologic iron losses, no known mechanisms of maintaining iron homeostasis have been identified, and losses appear to be related mainly to cell exfoliation (2).

In general, the causes of iron deficiency are: i. insufficient dietary iron intake; ii. reduced absorption of iron in the intestines; and iii. the most common, blood loss. Iron supplementation is not only needed in conditions of absolute deficiency, but evidence has shown an important role of iron in the trophism and function of the heart muscle in cardiac failure, even in the presence of normal or high iron stores (3). Thus, it is not surprising that one of the first complaints of iron deficiency is tachycardia.

As mentioned above, the leading cause of iron deficiency is acute or chronic blood loss, and the population bearing the highest risk for developing iron depletion is women of fertile age(4-6). This type of iron deficiency is often insidious as women have great difficulty in assessing the amount of their menstrual losses (7-9), and they may lose abnormally high amounts of blood during each menstruation period without being aware that the loss is excessive. In addition, neither the duration of menses nor the number of pads used is indicative of the actual blood loss (7). In the clinic, there are two main reasons why it is difficult to discern between 'normal' and 'abnormal' menstrual blood loss: firstly, the definition of 'normal' loss is largely subjective (7); and secondly, there are no simple and easily reproducible methods to measure blood losses. This explains why most cases of iron-deficient anemia in women with longstanding menorrhagia are diagnosed after long periods of iron-deficient erythropoiesis (10).

Iron requirements in women of fertile age are currently considered to be 15-20 $\mathrm{mg}$ /day (6). However, on average, only $10 \%$ of dietary iron is absorbed. Furthermore, there is substantial variation in the level of iron absorption due to a number of interfering factors, including the type of dietary iron (heminic, non-heminic) and elements in the diet either favoring (vitamin C) or impairing (phytates, tannins, polyphenols) iron absorption (11).

Knowledge of actual iron loss in women with 'normal' and 'abnormal' menstrual blood loss is scanty (9), and the relationship between iron loss and total menstrual loss is poorly understood, especially in women with menorrhagia. We believe that a detailed understanding of this relationship is important in order to prevent the development of iron-deficient anemia in this very frequent clinical setting. Therefore, we have evaluated total and day-by-day menstrual and iron losses on a large cohort of women with clearly defined 'normal' menstrual losses compared to a cohort of women with menorrhagia of various origins.

\section{Methods}

\section{Subjects}

A sample of 105 Caucasian women was prospectively enrolled: 84 had 'normal' menses and 21 had menorrhagia. The latter were referred to our clinic for an inherited or acquired bleeding disorder: 3 on oral anticoagulants for deep vein thrombosis, 3 with severe autosomally inherited bleeding disorders, 10 with dysfunctional uterine 
bleeding, and 5 with fibromyoma. All 105 women were born in Italy, were aged 20-45 years, and were recruited between January 2007 and October 2011 in three hospitals (Universities of L'Aquila and Palermo Academic Hospitals, and Research Hospital of S. Giovanni Rotondo). None of the women was pregnant or breastfeeding, none was taking oral contraceptive therapy for at least 3 months before enrollment, and none had medicated or non-medicated intra-uterine devices. Women with menorrhagia were diagnosed based on an internationally accepted parameter, MBL (12). The women with menorrhagia were exempt of treatments (i.e. hormone therapies, antifibrinolytics or replacement for their clotting defects). All subjects enrolled were administered a detailed general health and gynecologic questionnaire proposed by the SIGO (Società Italiana di Ginecologia ed Ostetricia) (13). In addition, a specific questionnaire produced for the calculation of the bleeding score in individuals with a 'mild bleeding disorder' (type I von Willebrand disease) (14) was administered in order to rule out a mild, unknown underlying bleeding disorder. Individuals were asked to provide information about their blood group and had their blood counts and serum ferritin (SF) assay measured 1 week to 1 day before the menstrual cycle under scrutiny. Informed consent was obtained from each patient enrolled in the current study

Measurement of blood loss (Menstrual Fluid Losses; MFL)

Participating women were asked to use the same brand and type of sanitary protection wears for the whole of their period, and to collect them using ad hoc labeled plastic bags. All women were instructed to collect all sanitary wears carefully to ensure minimal loss of menstrual flow in the toilet. All of the women used pads, except one woman who used tampons (internal sanitary wear). Participating women were asked to vacuum seal the plastic bags containing used wears promptly or at most within 2 hours, and to complete the required information (ID number, date and hour of collection) on the sticker identifying each bag. The hour of the first wear discharge and sealing was considered to be the start time of the menstrual cycle. One dry sanitary wear (blank) of the same brand (and package) was also vacuum-sealed.

At the end of each menstrual cycle, collected bags were sent to the Coordinator Center (Internal Medicine and Hematology Department, "San Salvatore" Hospital, L'Aquila). Bags were weighted on a scale sensitive to $1 \mathrm{~g}$, and weights were recorded after subtraction of the blank. Weights represented the Menstrual Fluid Losses (MFL). In a preliminary study, vacuum sealing was shown to maintain the weight and the chemical properties of the discharges effectively for up to 4 weeks (data not shown).

\section{Measurement of iron loss (Menstrual Iron Losses; MIL)}

Hematin extraction was conducted using a modification of the Alkaline Hematin Method (AHM) technique $(12,15)$. Standard spectrophometric analysis (Optical Density [OD]) was used to determine the concentration of hematin in each wear sample, using a standard curve obtained by diluting porcine hematin (Sigma-Aldrich, S. Louis, MO, USA). The following formula was applied to calculate hematin concentration $(\mu \mathrm{M})$ : OD hematin /0.0374)+0.02. Menstrual Iron Losses (MIL, $\mu \mathrm{g} / \mathrm{dl}$ ) were then calculated according the following formula: $\mathrm{MIL}=$ hematin concentration $(\mu \mathrm{M}) \times 55.85 / 10$. For each vacuum-sealed wear, the following results were obtained: 1. MFL in $\mathrm{ml}$ of fluid discharge (blood and secretions) measured by the weight and converted in $\mathrm{ml}$ by multiplying each figure by 1.025 , a conversion factor intermediate 
between the specific weight of blood and lymph; 2. MIL (iron losses) in $\mu \mathrm{g}$. Iron losses per day were calculated by dividing total losses by 28 (6).

\section{Statistical analysis}

The data description was based on standard measures of the distribution, such as median and mean for the position parameter and range and standard deviation (SD) for the variability. The normality of the parameter distribution was evaluated through the Kolmogorov Smirnov test. To evaluate parameter linear relations, we also calculated Pearson test and performed regression analysis. To compare median values of different groups, we used the Kruskal-Wallis test when the normality was rejected. Correlation analysis between sets of data was performed. Where useful, the confidence interval $(\mathrm{CI})$ of the test value and the $\mathrm{p}$-value $(\mathrm{p})$ were provided. Analyses were conducted using the medcalc software version 7.4.1.2 (http:www.medcalc.be).

This study protocol (MEVA-1 and MEVA-3) was first approved by the institutional review board (IRB) of the Istituto Di Ricovero e Cura a Carattere Scientifico (IRCCS) San Giovanni Rotondo, and subsequently by the IRBs of the University of L'Aquila and Palermo, and was registered on clinicaltrial.gov (NCT01276964). The study has been been performed in accordance with the ethical standards laid down in the 1964 Declaration of Helsinki and its later amendments.

\section{Results}

\section{Baseline demographic and clinical data}

Table 1 shows the demographic and clinical data pertaining to the 84 women with 'normal' menses and the 21 women with menorrhagia.

Women with 'normal' menses $(n=84)$ : Participants' median age was 28 years, and median body mass index (BMI) was $21 \mathrm{~kg} / \mathrm{m}^{2}$. Median menstruation duration was 5 days, median interval between cycles was 28 days , median age at menarche was 12.0 years , median hemoglobin $(\mathrm{Hb})$ level was $13.4 \mathrm{~g} / \mathrm{dl}$, and median SF level was 29 ng/L (Table 1).

Women with menorrhagia $(n=21)$ : All women with menorrhagia failed to meet the requirements needed to define normally menstruating women. Menstrual blood losses (MBL), preliminarily calculated with the $\operatorname{AHM}(7,12)$, were $>80 \mathrm{ml}$ in every case (data not shown). The median age of women with menorrhagia was 33.5 years. BMI was not available. Median menstruation duration was 6 days and the median interval between cycles was 28 days. Median age at menarche was 12.0 years. Median $\mathrm{Hb}$ level was $10.9 \mathrm{~g} / \mathrm{dl}$ and median SF level was $5.7 \mathrm{ng} / \mathrm{L}$ (Table 1).

\section{Menstrual and iron losses}

Table 2 compares data regarding MFL and MIL in both cohorts. Correlations between MIL and blood Hb, SF and MFL, both in 'normal' women and women with menorrhagia are reported in Table 3. Furthermore, Table 3 reports correlation between $\mathrm{SF}$ and MFL both in 'normal' women and women with menorrhagia. Figure 1 compares blood $\mathrm{Hb}$ and SF levels in normal women and women with menorrhagia. Figures 2 and 3 show total and day-by-day losses (MFL and MIL alike). 


\section{Discussion}

This study recruited a large number of women with either normal menses or menorrhagia to assess and compare menstrual and iron losses. Data showed that women with menorrhagia have a 5-6-fold higher loss of iron compared to women with normal menses, with a good correlation between the magnitude of blood loss (MFL) and iron loss (MIL) in both cohorts. In contrast, blood levels of $\mathrm{Hb}$ and SF do not predict losses.

Study participants did not receive any treatment commonly recognized to be capable of modifying menses duration or intensity. Of note, no contraceptives of any kind were prescribed, nor were devices in place. This is important as anovulatory hormones (in the contraceptive pill) are known to reduce menstrual losses by half (16), and intrauterine devices may actually double menstrual losses (17). In women with menorrhagia, MBL exceeded $80 \mathrm{ml}$ in every case (12), a figure that corresponds to $189 \mathrm{ml}$ of MFL.

We used two main approaches to evaluate menstrual discharge: a standardized pad weight assay method combined with a biochemical method that focused on the hematin concentration measurement after elution from the sanitary wears. The first approach (MFL) was indicative for blood and secretions discharged during the menses, and the second (MIL) was used to calculate directly the iron lost during the cycle.

To the best of our knowledge, this is the first time that iron losses have been directly measured. A historic study (18) evaluated radio-iron (59Fe) kinetics in women with menorrhagia, and showed a sharply increased absorption and a rapid disappearance from the bloodstream. However, in this previous study, the evaluation of iron lost with menses was not performed.

In the two cohorts in our study, day-by-day menstrual losses were notably different in terms of duration and daily distribution: in women with 'normal' menses, most of the blood losses were on days 1-3, whereas in women with menorrhagia, the peak blood loss was on day $2(\approx 30 \%$ ), with a gradual and slow decrease until day 7 (Figure 2,3 ).

The comparative analysis between normally menstruating women and women with menorrhagia evidenced a clear-cut difference between the two cohorts, both in terms of total losses (MFL) and of iron losses (MIL). The difference is also evidenced in Figure 1 where blood $\mathrm{Hb}$ and SF levels were compared in the two cohorts. This can be ascribed to the strict method used to assess the range of 'normality'. On average, women with menorrhagia had a 5-to-6-times higher iron loss per cycle (range 3-16) in comparison to women with normal menses, and very few women with menorrhagia overlapped the normal value distribution for MFL $(n=2)$ and MIL $(n=5)$.

The present study showed that the correlation between MFL and MIL was very good, both in women with normal menses and in women with menorrhagia (Table 3 ), with about $65 \mathrm{ml}$ of MFL accounting for $1 \mathrm{mg}$ of iron lost (CI 930-1070 $\mu \mathrm{g}$ ). In contrast, correlations between blood measurements ( $\mathrm{Hb}$ and $\mathrm{SF}$ ) and menstrual fluid measurements were fairly poor (Table 3), indicating that it is difficult to predict iron losses solely on the basis of blood parameters. However, a fairly good correlation was found between SF and MFL (in the cohort with menorrhagia only). Nevertheless, 
these data show that the process to reach depleted iron stores is highly variable and may be of long duration, depending on both the baseline iron stores and the amount of iron lost per cycle. Other factors that may delay the identification of iron deficiency status include the choice of laboratory methods, the cut-off values used, the presence of other disorders, and the subjective recognition of related symptoms.

Our data clearly showed that iron loss in women with menorrhagia was significantly higher than in the control group (Table 2). This is an expected finding, although its magnitude had not previously been reported. All of the women enrolled with menorrhagia had ferritin levels indicating that iron store depletion had already taken place; however, none of them, when referred to us, was taking iron therapy. Iron losses of the magnitude shown, and the fact that even women with normal menstruations have seldom replenished iron stores, suggests that the development of iron-deficient anemia is very likely in the short term. However, most of the time, women are referred to the gynecologist or the hematologist when iron depletion is well advanced. Instead, it would be important, from a clinical standpoint, to ascertain the abnormality of the menstrual losses as soon as possible when iron-deficient erythropoiesis has not yet developed. Based on these data, we suggest that treatment to correct iron-deficient erythropoiesis and replenish iron stores should be instituted as soon as possible, although the main obstacle is a late diagnosis of menorrhagia. Because of the poor predictive value of blood parameters concerning the amount of iron lost per menstrual cycle, even an indirect, quantitative evaluation of the iron lost with menses may provide elements useful to assess the risk of developing irondeficient anemia in individual patients.

A possible limitation of this study is that we tested only one menstrual cycle. However, it is widely accepted that menstrual losses in individual women are fairly constant throughout their fertile age (19), a fact for which a genetic control has been proposed (20).

In conclusion, this study demonstrates a correlation between the magnitude of menstrual blood loss (MFL) and iron loss (MIL), and a greater loss of iron in women with menorrhagia compared to women with normal menses. There is a lack of correlation between blood parameters ( $\mathrm{Hb}$ and $\mathrm{SF}$ ) with losses. These findings highlight the importance of early diagnosis of menorrhagia, assessment of iron loss, and prompt treatment to prevent iron-deficient anemia. 


\section{Acknowledgements}

Authors would like to thank all physicians and women involved in this study Authors contribution

Mariasanta Napolitano and Guglielmo Mariani ideated the work and wrote the paper. Alberto Dolce performed the statistical analysis of results. Mariasanta Napolitano, Elvira Grandone, Sergio Siragusa, Assunta Orecchioni enrolled patients and critically revised the results. Giuseppe Celenza and Maria Grazia Perilli supported the laboratory research. Gaspare Carta contributed to the study design and enrolment. Guglielmo Mariani critically revised all the research process. All the authors have significantly contributed to the research, they have read and approved the submitted version of the article.

In addition to institutional funds, an unrestricted grant from Novo Nordisk helped to conduct the study.

\section{Conflict of interest}

The authors declare that they have no conflict of interest 


\section{References}

1. Finberg KE. Unraveling mechanisms regulating systemic iron homeostasis. Hematology Am Soc Hematol Educ Program 2011;2011:532537.

2. Zhang AS, Enns CA. Molecular mechanisms of normal iron homeostasis. Hematology Am Soc Hematol Educ Program 2009;2009:207-214.

3. Okonko DO, Mandal AK, Missouris CG, Poole-Wilson PA. Disordered iron homeostasis in chronic heart failure: prevalence, predictors, relation to anemia, exercise capacity, and survival. J Am Coll Cardiol 2011;58:12411251.

4. Charlton RW, Bothwell TH. Definition, prevalence and prevention of iron deficiency. Clin Haematol 1982;11:309-325.

5. Cook JD. Clinical evaluation of iron deficiency. Semin Hematol 1982;19:6-18.

6. Hallberg L, Rossander-Hultén L. Iron requirements in menstruating women. Am J Clin Nutr 1991;54:1047-1058.

7. Fraser IS, McCarron G, Markham R. A preliminary study of factors influencing perception of menstrual blood loss volume. Am J Obstet Gynecol 1984;149:788-793.

8. Fraser IS, McCarron G, Markham R, Resta T. Blood and total fluid content of menstrual discharge. Obstet Gynecol 1985;65:194-198.

9. Warner PE, Critchley HO, Lumsden MA et al. Menorrhagia II: is the 80$\mathrm{mL}$ blood loss criterion useful in management of complaint of menorrhagia? Am J Obstet Gynecol 2004;190:1224-1229.

10. Warner PE, Critchley HO, Lumsden MA et al. Menorrhagia I: measured blood loss, clinical features, and outcome in women with heavy periods: a survey with follow-up data. Am J Obstet Gynecol 2004;190:1216-1223.

11. Strong J. Hematinic deficiencies. In: Pavord S, Hunt B, editors. The Obstetric Hematology Manual. Cambridge University Press; 2010:13-27

12. Shaw ST Jr, Aaronson DE, Moyer DL. Quantitation of menstrual blood loss-further evaluation of the alkaline hematin method. Contraception 1972;5:497-513.

13. Società Italiana di Ginecologia e Ostetricia (SIGO), www.sigo.it.

14. Rodeghiero F, Castaman G, Tosetto A et al. The discriminant power of bleeding history for the diagnosis of type I von Willebrand disease: an international, multicenter study. J Thromb Haemost 2005;3:2619-2626.

15. Hallberg L, Nilsson L. Determination of menstrual blood loss. Scand J Clin Lab Invest 1964;16:244-248.

16. Nilsson L, Sölvell L. Clinical studies on oral contraceptives-a randomized, doubleblind, crossover, study of 4 different preparations (Anovlar mite, Lyndiol mite, Ovulen, and Volidan). Acta Obstet Gynecol Scand 1967;46:Suppl 8:1-31. 
17. Guillebaud J, Bonnar J, Morehead J, Matthews A. Menstrual blood-loss with intrauterine devices. Lancet 1976;1:387-390.

18. Price DC, Forsythe EM, Cohn SH, Cronkite EP. The study of menstrual and other blood loss, and consequent iron deficiency, by $\mathrm{Fe}^{59}$ whole-body counting. Can Med Assoc J 1964;90:51-54.

19. Hallberg L, Nilsson L. Constancy of individual menstrual blood loss. Acta Obstet Gynecol Scand 1964;43:352-359.

20. Rybo G, Hallberg L. Influence of heredity and environment on normal menstrual blood loss. A study of twins. Acta Obstet Gynecol Scand 1966;45:389-410. 


\section{Figure legends}

Figure 1-Title: Serum ferritin and blood Hemoglobin levels in women with normal menses and women with menorrhagia

Legend: $\mathrm{SF}=$ Serum ferritin, $\mathrm{Hb}=$ Hemoglobin levels (Alberto effettivamente credo abbiamo invertito i nomi degli assi, no? E cioè in ordinata con i livelli da 7 a 16 dovremmo riferirci a $\mathrm{Hb}(\mathrm{g} / \mathrm{dL})$, mentre in ascissa con i livelli da 0 a 120 dobbiamo riportare la ferritina sierica SF in (ng/100ml))

Figure 2- Title $=$ Mean Fluid Losses in normal and menorrhagic women

Legend $=$ MFL $=$ Mean Fluid Losses, expressed as percentage of total during the different days of menses. Boxes indicate mean and whiskers Standard Deviation(?)

Figure 2- Title= Mean Iron Losses in normal and menorrhagic women

Legend $=$ MIL=Mean Iron Losses, expressed as percentage of total during the different days of menses. Boxes indicate mean and whiskers Standard Deviation(?) 